\title{
Hemodiálisis: ¿Cuánto sabemos de los fármacos relacionados con el metabolismo mineral?
}

\author{
Isidro Sánchez Villar * - Víctor Lorenzo Sellares**
}

*Enfermero

**Nefrólogo

Servicio de Nefrología del Hospital Universitario de Canarias. Sta. Cruz de Tenerife.

\section{Resumen}

Las complicaciones derivadas de la alteración del metabolismo óseo-mineral tienen un gran impacto sobre los pacientes con enfermedad renal crónica terminal. El arsenal terapéutico usado para prevenir y resolver estas complicaciones ha evolucionado. Estos fármacos -entre otros- son supervisados 0 administrados por el personal de enfermería. Los profesionales de enfermería tienen una importante labor de educación sanitaria y su conocimiento puede ayudar a aclarar las dudas de los pacientes y mejorar su adherencia terapéutica.

El objetivo de este estudio ha sido valorar el grado de conocimiento que los profesionales de enfermería nefrológica tienen de los fármacos utilizados en la corrección de las alteraciones del metabolismo mineral que administran o supervisan en la sesión de diálisis.

Se realizó un estudio descriptivo observacional mediante una encuesta de seis preguntas relacionadas con variables sociodemográficas y ocho tipo test, realizada en el XXXV Congreso Nacional de la SEDEN.

Correspondencia:
Isidro Sánchez Villar
Servicio de Nefrología
Hosp. Universitario de Canarias
C/. Ofra s/n
38320 La Laguna
Tenerife
isvillar@ya.com

Fueron entrevistados 72 profesionales. El 36\% desempeñaba su labor en Unidades Periféricas. Sólo el $43 \%$ trabajaba en centros donde se administraba medicación oral relacionada con el fósforo: $57 \%$ en el caso de los centros hospitalarios frente al $9 \%$ de los centros periféricos $(p=0,002)$.

La puntuación media, sobre un máximo de 8 puntos, fue de 3,32 . Solamente el $17 \%$ respondió acertadamente los $2 / 3$ de las preguntas. No existió relación entre la puntuación obtenida y la edad de los encuestados, aunque los encuestados mayores de 44 años $(p=0.062)$ presentan puntuaciones más bajas.

La puntuación obtenida por los profesionales de centros hospitalarios $(3,41 \pm 2,03)$ fue ligeramente superior a la obtenida por los profesionales de centros periféricos $(3,15 \pm 2,22)$ aunque sin significación estadística $(p=0,62)$. Tampoco encontramos asociación entre el tiempo como profesionales de enfermería, el tiempo desempeñado en unidades de diálisis y la puntuación obtenida.

Como conclusión podemos decir que son pocos los centros de diálisis que administran quelantes durante las sesiones, y que hay que mejorar sustancialmente la información sobre estos medicamentos que supervisa y administra enfermería y sobre los que los pacientes reclaman ayuda en forma de información. 


\section{PALABRAS CLAVE: \\ - ENFERMERÍA \\ - DIÁLISIS \\ - ADHERENCIA TERAPÉUTICA \\ - CONOCIMIENTO FARMACOLÓGICO \\ - METABOLISMO MINERAL}

\section{Haemodialysis: How much do we know about the drugs related to mineral metabolism?}

\section{Abstract}

The complications deriving from the alteration of the bone mineral metabolism have a considerable impact on patients with terminal chronic renal disease. The therapeutic weapons used to prevent and overcome these complications have evolved. These drugs -among others- are supervised or administered by nursing staff. Nurses have an important health education task and their knowledge can help to clear up patients' doubt and improve their adherence to treatment.

The aim of this study was to evaluate the degree of knowledge that nephrology nursing professionals have of the drugs used in the correction of mineral metabolism alterations administered or supervised by them in the dialysis session.

An observational descriptive study was carried out using a survey comprising six questions related to socio-demographic variables and eight multiple choice questions, drawn up in the 35th National Congress of SEDEN.

A total of 72 professionals were interviewed. of these, 36\% worked in Peripheral Units. Only 43\% worked in centres where oral medication related to phosphorous was administered: $57 \%$ in the case of hospitals compared to $9 \%$ in the peripheral centres $(p=0.002)$.

The mean score, out of a maximum of 8 points, was 3.32. Only $17 \%$ answered $2 / 3$ of the questions correctly. There was no relationship between the score obtained and the age of the interviewees, although interviewees aged over $44(p=0.062)$ had lower scores.
The score obtained by professionals in hospitals $(3.41 \pm 2.03)$ was slightly higher than the score obtained by professionals from peripheral centres $(3.15 \pm 2.22)$ although not statistically significant $(p=0.62)$. Nor did we find an association between the time in the nursing profession, the time spent in dialysis units and the score obtained.

As a conclusion we can state that there are few dialysis centres that administer chelants during sessions, and substantial improvement is needed in relation to information on these medications which are supervised and administered by nursing staff and on which patients request help in the form of information.

\section{KEY WORDS:}

- NURSING, DIALYSIS

- TREATMENT ADHERENCE

- DRUG KNOWLEDGE

- MINERAL METABOLISM

\section{Introducción}

Desde que en 1957 se realizó la primera hemodiálisis con carácter de continuidad ${ }^{1}$ hasta el día de hoy, el perfil de los pacientes ha cambiado sustancialmente. La enfermedad renal crónica terminal (ERCT) actualmente afecta sobre todo a la población de edad más avanzada, con un incremento en el riesgo a medida que aumenta ${ }^{2}$. Este incremento en la edad se acompaña de un aumento de la comorbilidad con implicaciones no sólo en la morbimortalidad, sino también sobre aspectos funcionales cotidianos ${ }^{3}$. En estos pacientes, las complicaciones derivadas de la alteración del metabolismo óseo-mineral tienen un gran impacto $0^{4,5,6,7}, \mathrm{y}$ en su prevención debe estar implicado todo el equipo sanitario. Paralelamente, tratando de prevenir y resolver estas complicaciones, el arsenal terapéutico ha evolucionado. Con frecuencia estos fármacos -entre otros- son supervisados o administrados por el personal de enfermería. En este contexto, los profesionales de enfermería tienen una importante labor de educación sanitaria y su conocimiento puede ayudar a aclarar las dudas de los pacientes y mejorar su adherencia 
terapéutica. Teniendo en cuenta estas consideraciones deberíamos preguntarnos, ¿cuál es el grado de advertencia de los profesionales de enfermería en el uso de estos fármacos?

Por eso nuestro objetivo ha sido examinar, mediante una encuesta, el grado de conocimiento de los fármacos utilizados en la corrección de las alteraciones del metabolismo mineral que los profesionales de enfermería nefrológica administran o supervisan en la sesión de diálisis.

\section{Material y método}

Se realizó un estudio descriptivo observacional mediante una encuesta (anexo 1) realizada en el XXXV Congreso Nacional de la Sociedad Española de Enfermería Nefrológico, celebrado del 7 al 10 de octubre del 2009 en Pamplona a una muestra aleatoria de 72 profesionales de Enfermería, el 8,5\% de los asistentes al Congreso.

La encuesta contenía seis preguntas relacionadas con variables sociodemográficas y ocho tipo test. Cada pregunta presentaba cinco posibles respuestas relacionadas con conocimientos de fármacos de manejo habitual en el contexto de las alteraciones del metabolismo mineral en la ERCT estadio 5D.

Las encuestas fueron realizadas por cinco profesionales de enfermería. El entrevistador entregaba el cuestionario, y se encargaba de anotar el número que daba por respuesta el entrevistado a cada una de las preguntas en una planilla que sólo contenía cuadros anexos a cada pregunta. Finalizado el proceso se recogía el cuestionario.

\section{Estadística:}

Las variables numéricas se expresaron como media y desviación estándar. Las variables cualitativas o categóricas se presentaron como proporciones o porcentajes. Para el análisis estadístico entre dos proporciones se utilizó el test Chi-cuadrado o la prueba exacta de Fisher si las frecuencias observadas eran pequeñas. Para el análisis de las variables en las que existían más de dos grupos se utilizaron test de análisis de varianza Anova. El estudio de relación entre variables cuantitativas se realizó mediante el cálculo de los coeficientes de correlación "r" de Pearson. Se consideró significativo

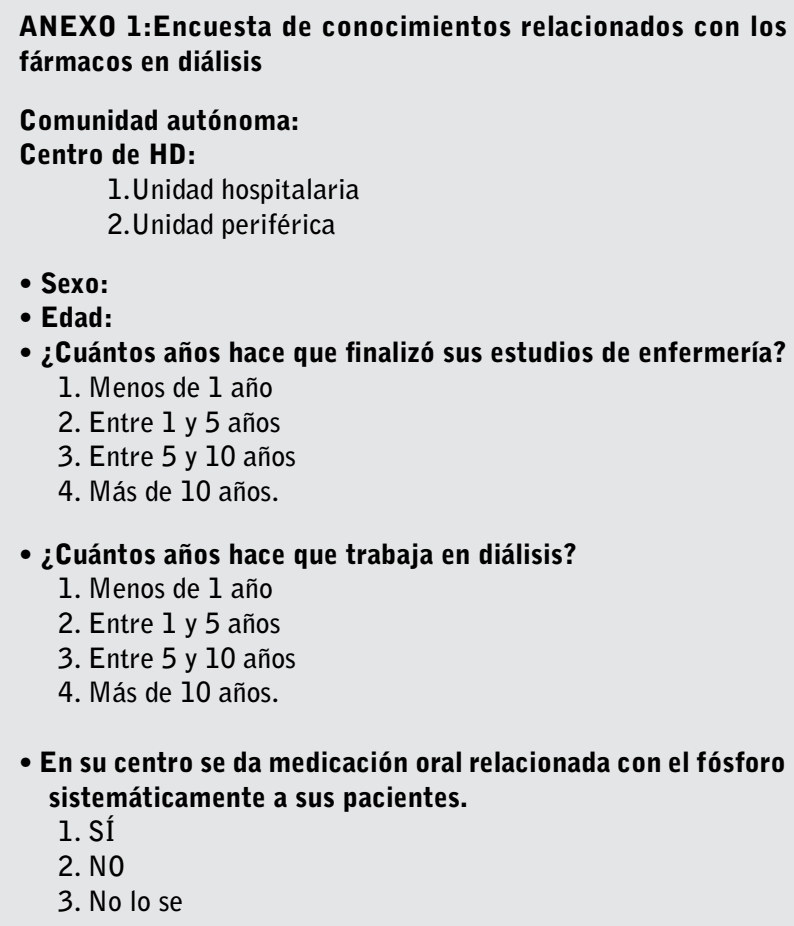

ANEXO 1:Encuesta de conocimientos relacionados con los fármacos en diálisis

Comunidad autónoma:

Centro de HD:

1.Unidad hospitalaria

2.Unidad periférica

- Sexo:

- Edad:

- ¿Cuántos años hace que finalizó sus estudios de enfermería?

1. Menos de 1 año

2. Entre 1 y 5 años

3. Entre 5 y 10 años

4. Más de 10 años.

- ¿Cuántos años hace que trabaja en diálisis?

1. Menos de 1 año

2. Entre 1 y 5 años

3. Entre 5 y 10 años

4. Más de 10 años.
- En su centro se da medicación oral relacionada con el fósforo sistemáticamente a sus pacientes.
1. SÍ
2. NO
3. No lo se

1. ¿Cuál de estos fármacos NO está relacionado con la corrección del metabolismo calcio-fósforo?

1. Hidróxido de aluminio.

2. MIRCERA

3. Cinacalcet (mimpara)

4. Carbonato cálcico.

5. No lo sé.

2. ¿Cuál de estos fármacos SI es un quelante del fósforo?

1. Bicarbonato sódico

2. Carbonato de lantano

3. Cinacalcet (mimpara)

4. Paricalcitol (zemplar)

5. No lo sé.

3. De estas afirmaciones relacionadas con los quelantes del fósforo, ¿Cuál considera que NO es correcta?

1. Los quelantes No deben darse en diálisis, porque se dializan, perdiendo su eficacia.

2. Los quelantes deben darse junto con las comidas.

3. Los quelantes son un grupo de medicamentos que se toman para reducir la absorción del fosfato.

4. Uno de los efectos secundarios más frecuente de los quelantes es la mala tolerancia gástrica.

5. No lo sé.

4. De estas afirmaciones relacionadas con el carbonato de lantano, ¿Cual considera que sí es correcta?

1. Su toma en HD es ineficaz (se dializa), y además puede disminuir la TA.

2. La dosis que aporta de calcio es muy superior a la del resto de los quelantes del fósforo.

3. El carbonato de lantano es un calcimimético.

4. Los comprimidos de carbonato de lantano deben triturarse previo a su ingesta.

5. No lo sé. 
5. De estas afirmaciones relacionadas con el cinacalcet (mimpara) ¿Cuál considera que Sí es correcta?

1. La EV es la vía más frecuente.

2. Es el más potente de los quelantes del fósforo.

3. Se usa para tratar el Hiperparatiroidismo secundario

4. En pacientes tratados con Cinacalcet (mimpara) deben monitorizarse los niveles de glucemia al menos una vez al mes.

5. No lo sé.

6. De estas afirmaciones relacionadas con el paricalcitol (zemplar) ¿Cuál considera que Sí es correcta?

1. Debe tomarse en todas las comidas.

2. Está indicado cuando los niveles de fósforo en sangre está por debajo de las cifras normales

3. Interfiere con la actividad de la heparina en diálisis, por lo que debe administrase finalizada la diálisis.

4. Se usa para tratar el Hiperparatiroidismo secundario.

5. No lo sé

7. De estas afirmaciones relacionadas con el calcitriol ¿Cuál considera que Sí es correcta?

1. Es la forma activa de la vitamina $D$

2. La mayor parte del fármaco es calcio, y por eso se utiliza para regular el calcio en sangre

3. Es un buen quelante del fósforo

4. Se usa para aumentar los niveles de fósforo en sangre

5. No lo sé

8. De estas afirmaciones en la ERC estadio 5 con tratamiento sustitutivo en HD. ¿Cuál considera que NO es correcta?

1. Los niveles recomendados de fosforemia son entre 2,7 y 5 $\mathrm{mg} / \mathrm{dl}$

2. La PTH debe mantenerse por encima de $800 \mathrm{pg} / \mathrm{ml}$

3. La calcemia debe situarse entre 8,4 y $9,5 \mathrm{mg} / \mathrm{dl}$

4. El valor recomendado del producto $\mathrm{Ca} \times \mathrm{P}$ debe ser menor de $55 \mathrm{mg} / \mathrm{dl}$

5. No lo se

un valor de $\mathrm{p}$ bilateral menor de 0,05. Para el análisis de los datos se utilizó el soporte informático SPSS 17.0. software (SPSS Inc, Chicago).

\section{Resultados}

De los 74 profesionales a los que se les propuso realizar la encuesta, 72 (97\%) aceptaron responderlo. Las características de la población encuestada se describen en la Tabla 1. En la Tabla 2 se describe la distribución por comunidades autónomas de las personas encuestadas.

De los 72 profesionales entrevistados, el 36\% desempeñaba su labor en Unidades Periféricas. Sólo el $43 \%$ de los encuestados trabajaban en centros donde se administraba sistemáticamente medicación oral relacionada con el fósforo a sus pacientes. Esta administración era del $57 \%$ en el caso de los centros hospitalarios frente al $9 \%$ de los centros periféricos $(p=0,002)$.

\begin{tabular}{|l|c|}
\hline Sexo & $78 \%$ mujeres \\
\hline $\bar{X}$ edad & $37 \pm 10$ años \\
\hline $\bar{X}^{\circ}$ de enfermería & $9 \pm 6$ años \\
\hline $\bar{X} \mathrm{t}^{\circ}$ enfermera en HD & $7 \pm 5$ años \\
\hline
\end{tabular}

Tabla 1. Características de la población

\begin{tabular}{|c|c|c|c|}
\hline & & Frecuencia & Porcentaje \\
\hline ह & ANDALUCIA & 4 & 5,6 \\
\hline$\overline{\equiv \equiv}$ & ARAGON & 3 & 4,2 \\
\hline 业 & BALEARES & 7 & 9,7 \\
\hline & CANARIAS & 9 & 12,5 \\
\hline & CASTILLA-MANCHA & 3 & 4,2 \\
\hline 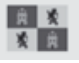 & CASTILLA Y LEON & 6 & 8,3 \\
\hline 豆 & CATALUÑA & 8 & 11,1 \\
\hline 붑를 & EUSKADI & 3 & 4,2 \\
\hline & GALICIA & 9 & 12,5 \\
\hline$\cdots$ & MADRID & 7 & 9,7 \\
\hline & MURCIA & 3 & 4,2 \\
\hline$=$ & NAVARRA & 5 & 6,9 \\
\hline 限 & VALENCIA & 5 & 6,9 \\
\hline 5 & Total & 72 & \\
\hline
\end{tabular}

Tabla 2. Distribución de encuestados por comunidades

En la Figura 1 se observa los porcentajes generales de respuestas correctas e incorrectas a las preguntas de conocimiento. A excepción de la pregunta 1 , que respondió correctamente el $67 \%$ de los encuestados, y la 5 con el $50 \%$, el resto de las preguntas no alcanza el $50 \%$ de respuestas correctas.

La puntuación media de las personas entrevistadas fue de 3,32 $\pm 2,14(0-8)$, sobre un máximo de 8 puntos posibles. 


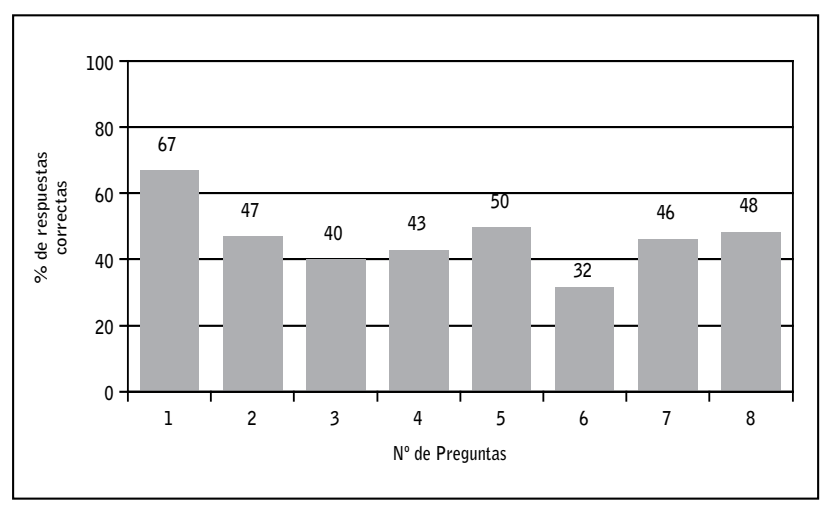

Figura 1: \% de respuestas correctas por pregunta

En la Figura 2 se observa la distribución de las puntuaciones en tres grupos. Es notable que solamente el $17 \%$ de los encuestados fuera capaz de responder acertadamente los 2/3 de las preguntas.

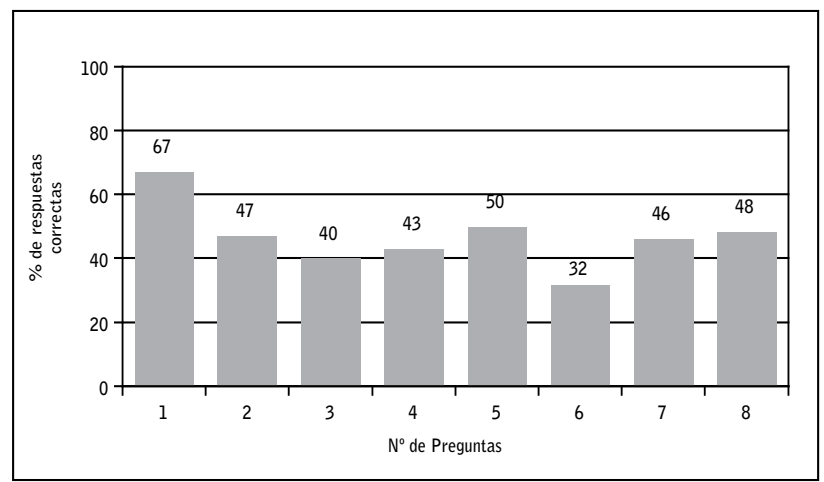

Figura 2: \% Distribución de puntuaciones

\section{Asociación entre conocimiento de fármacos y perfil profesional}

Analizado el promedio de las puntuaciones obtenidas por los profesionales de centros hospitalarios $(3,41 \pm 2,03)$ se observa que fue ligeramente superior a las obtenida por los profesionales de centros periféricos $(3,15 \pm 2,22)$ aunque sin significación estadística $(p=0,62)$.

No existió relación entre la puntuación obtenida y la edad de los encuestados aunque hay una tendencia a presentar puntuaciones más bajas de los encuestados mayores de 44 años ( $p=0.062$ ). Tampoco observamos una relación definida entre el tiempo como profesionales de enfermería y la puntuación obtenida $((p=0.365)$ y entre el tiempo desempeñando la labor en hemodiálisis y puntuación $(p=0.365)$. Sin embargo hay un patrón similar a la edad, de manera que obtienen peor puntuación los profesionales con más tiempo en diálisis.

\section{Discusión:}

Considerando la relevancia que para los pacientes en programa de hemodiálisis tienen los problemas derivados de las alteraciones del metabolismo mineral ${ }^{(4,5,6,7)}$, el manejo que realiza habitualmente el colectivo de enfermería de fármacos diseñados para prevenir y tratar estas complicaciones, y su importante labor de educadores sanitarios, parece evidente que la media de 3,4 puntos sobre 8 posibles es una puntuación francamente mejorable.

Se escogió como punto de corte, para considerar un conocimiento aceptable, el tercer cuartil de puntuación, es decir, más de 5 respuestas acertadas $(62 \%$ de aciertos). Observamos que tan sólo el $18 \%$ alcanza al menos esa puntuación. Solamente el 2,8\% marca correctamente todas las preguntas, y es Ilamativo que el $4,2 \%$ obtiene 0 respuestas acertadas.

No hemos encontrado estudios publicados acerca del conocimiento de fármacos en hemodiálisis que nos puedan orientar sobre las posibles causas de tan escaso conocimiento. Aun así parece incuestionable que debemos reflexionar sobre ello.

Si observamos los resultados podemos apreciar que aunque no significativa ( $p=0.14$ ), existe diferencia de puntuación entre los profesionales que en sus centros dan fármacos relacionados con el metabolismo mineral a los pacientes $(3,74)$ frente a los que no lo hacen $(3,00)$. También hay una sutil diferencia de puntuación $(p=0,62)$ entre los profesionales del ámbito hospitalario $(3,41)$ frente a los que trabajan en centros periféricos $(3,15)$. Hay que considerar que los fármacos orales relacionados con el fósforo se administran en el $57 \%$ de los centros hospitalarios frente al $9 \%$ de los centros periféricos, por lo que el factor relevante tendría más relación con la familiaridad en administrar estos fármacos que con el factor centro. Aun así no justifica la baja puntuación.

Desde que a finales de los años ochenta aparecieron la EP0 y el tampón bicarbonato, la técnica de hemodiálisis se ha estabilizado. Podríamos decir que la técnica como tal es rutinaria, y que los avances que se producen se integran perfectamente en esta rutina. ¿Es entonces la hemodiálisis una técnica rutinaria que no precisa integrar nuevos conocimientos, al menos referidos al metabolismo mineral? 
Si observamos la edad de los encuestados vemos que se produce una curva de puntuación, la puntuación mejora con la edad hasta el tercer cuartil (36 a 44 años) donde alcanza la máxima puntuación y paradójicamente decrece significativamente $(p=0,03$ en el último cuartil (>44 años)). La misma curva se observa con la puntuación y el tiempo como profesionales de enfermería y el tiempo como profesionales en HD. Parece que este patrón encaja con el planteamiento de la pregunta: el tiempo genera rutina.

Diferentes estudios refieren que una adecuada información sobre los medicamentos del personal de enfermería mejora el cumplimiento terapéutico de los pacientes $8,9,10,11,12,13$. Sin embargo en ocasiones escuchamos en las salas de diálisis indicaciones erróneas: "No tome ninguna pastilla en diálisis porque la "máquina" las dializa (incluyendo quelantes)", "el calcijex es calcio", "las pastillas para el fósforo mejor tomarlas una hora después de las comidas", etc. Con todo esto, parece que el diagnóstico podría acercarse a la inadecuada formación en las unidades de diálisis en esta materia, sumada con un cierto desinterés por parte del personal con mayor edad y tiempo en las unidades que no se justifica por situaciones de Burnout ${ }^{14,15,16}$.

El conocimiento del personal de enfermería, al menos, en relación con los fármacos relacionados con el metabolismo mineral tiene un claro espacio para la mejora. Esta mejora pueda darse, probablemente con una mejor predisposición a la formación y una mayor intercomunicación entre estamentos sanitarios e incluso farmacéuticos, no olvidemos que los nefrólogos prescriben los fármacos, pero las enfermeras los administran. El perfil actual del paciente renal se relaciona con una persona anciana, polimedicada y con un grado de dependencia elevado, con dificultades evidentes para cumplir adecuadamente su tratamiento farmacológi$\mathrm{CO}^{17,18,19}$. Si hablamos de datos relacionados con la falta de adherencia terapéutica podemos ver series referidas por algunos autores $9,20,21$ entre el 40 y $50 \%$ en diálisis. Consideramos que es muy importante que enfermería se implique en la labor de educación hacia el paciente de forma global. Consideramos que esto puede tener un impacto importante en la mejora de estos porcentajes; por lo que es imprescindible que las enfermeras posean las herramientas conceptuales necesarias.

Como conclusión podemos decir que son pocos los centros de diálisis que administran quelantes durante las sesiones, y que hay que mejorar sustancialmente la información sobre estos medicamentos que supervisa y administra enfermería y sobre los que los pacientes reclaman ayuda en forma de información.

\section{Agradecimientos}

Agradecemos a las enfermeras Rosa Amelia Dorta, Ana Esther Martín, Pilar Marrero y al enfermero Oscar Álvarez, del Hospital Universitario de Canarias de Tenerife su inestimable ayuda en la realización de las encuestas y a todas las enfermeras y enfermeros que se prestaron a responderlas.

Recibido:Diciembre 2010

Revisado:Diciembre 2010

Modificado: Enero 2011

Aceptado: Enero 2011

\section{Bibliografía}

1. Stewart Cameron, J. Breve historia de la hemodiálisis (1850-1970). Hombres, materiales e ideas. En: Tratado de Hemodiálisis. Barcelona: Editorial Médica Jims. SL; 2006. p. 19.

2. Diálisis y Trasplante 2007 Registro Español de Enfermos Renales Informe preliminar. [citado 2010 Ene 15] Disponible en: http://www.senefro. org/modules/subsection/files/informe_preliminar_ reer_2007_sen_2008.pdf?check_idfile=3558.

3. Aladrén M. J., Pérez y Pérez J., Azuara M, Berisa F. Hemodiálisis en pacientes de edad avanzada. Estudio multicéntrico de las sociedades aragonesa y norte de nefrología. Nefrología 1999. Vol. XIX. Núm. 1. 39-48.

4. V. Lorenzo, M. Rodríguez Portillo, R. Pérez García y J. B. Cannata. De la osteodistrofia renal a las alteraciones del metabolismo óseo y mineral asociado a la enfermedad renal crónica: evolución de un concepto. Nefrología 2007; 5:527-533.

5. Block GA, Raggi P, Bellasi A y cols. Mortality effect of coronary calcification and phosphate binder choi- 
ce in incident hemodialysis patients. Kidney Int 2007; 71(5): 438-41.

6. Q Qunibi WY. Consequences of hyperphosphatemia in patients with endstage renal disease (ESRD). Kidney Int 2004; 90:S8-S12.

7. Block GA, Hulbert-Shearon TE, Levin NW et al. Association of serum phosphorus and calcium $\mathrm{x}$ phosphate product with mortality risk in chronic hemodialysis patients: a national study. Am J Kidney Dis 1998; 31: 607-617.

8. Ortigosa Barriola, A, Sesmero Ramos, C, Velayos González, M ${ }^{a}$ P, Portolés Pérez, J. Actuación de enfermería relacionada con pacientes en diálisis peritoneal y tratamiento con cinacalcet. Rev Soc Esp Enferm Nefrol 2007; 10 (3): 173-177.

9. Ochando García A, Royuela García C, Hernández Meca $M^{a} E$, Lorenzo Martínez S, Paniagua Fernando. Influencia de la satisfacción de los pacientes de una unidad de diálisis en la adherencia terapéutica. Rev Soc Esp Enferm Nefrol [revista en la Internet]. 2008 Dic [citado 2010 Feb 15]; 11(4): 271-276. Disponible en: http://scielo.isciii.es/scielo.php?script=sci_ arttext\&pid=S1139-13752008000400005\&lng=es.

10. Kammerer J, Garry G, Hartigan M, Carter B, Erlich L. Adherence in Patients On Dialysis: Strategies for Success Nephrolog. nursing journal 2007 SepOct;34(5):479-86.

11. Manns BJ, Taub K, VanderStraeten C, Jones H, Mills $C$, Visser $M$, et al. The impact of education on chronic kidney disease patients' plans to initiate dialysis with self-care dialysis: A randomized trial. Kidney International 2005;68(4):1777-1783.

12. Costantini L. Compliance, adherence, and selfmanagement: is a paradigmshift possible for chronic kidney disease clients? The CANNT Journal 2006;16(4):22-26.

13. Patient education and the nursing process: meeting the patient's needs. Wingard R. Nephrol Nurs J. 2005 Mar-Apr;32(2):211-4; quiz 215.

14. Del Campo M, Fernández-Repeto E, Martínez J, Rojas A. El síndrome de quemarse por el trabajo (burnout) en los profesionales de Enfermería de las unidades de diálisis de la provincial de Cádiz. XXIV Congreso Nacional de la Sociedad Española de Enfermería Nefrológica 1999. Valencia, España [en línea]1999. [citado 2010 Feb 15] ; Disponible en: http://www.seden.org.

15. Flynn L, Thomas-Hawkins C, Clarke SP Organizational traits, care processes, and burnout among chronic hemodialysis nurses. West J Nurs Res. 2009 Aug;31(5):569-82. Epub 2009 Mar 6.

16. Gardner JK, Thomas-Hawkins C, Fogg L, Latham CE. The relationships between nurses' perceptions of the hemodialysis unit work environment and nurse turnover, patient satisfaction, and hospitalizations. Nephrol Nurs J. 2007 May-Jun;34(3):271-81; quiz 282.

17. Leal Hdez M,Abellán Alemán J,Casa Pina MT,Martínez Crespo J. Paciente polimedicado: ¿conoce la posología de la medicación?, ¿afirma tomarla correctamente?. Aten Primaria 2004;33:451-456.

18. Abril Sabater $M^{a}$ Dolores, Iglesias Sanjuán Ruben, Jerez Garcia Almudena, López Parra María J., Mañé Buixó Núria, Yuste Jiménez Elios. Aplicación de una técnica cualitativa para la elaboración de un plan de atención al paciente pluripatológico en hemodiálisis. Rev Soc Esp Enferm Nefrol [revista en la Internet]. 2004 Dic [citado 2010 Feb 17]; 7(4): 67-71. Disponible en: http://scielo.isciii.es/scielo.php?script=sci_ arttext\&pid=S1139-13752004000400005\&lng=es.

19. Contreras Abad M $\mathrm{M}^{\mathrm{a}}$ Dolores, Rivero Arellano $\mathrm{M}^{\mathrm{a}}$ Flora, Jurado Torres $\mathrm{M}^{\mathrm{a}}$ Jesús, Crespo Montero Rodolfo. Perfil actual del paciente en hemodialisis hospitalaria: Análisis de sus necesidades. Rev Soc Esp Enferm Nefrol [revista en la Internet]. 2004 Mar [citado 2010 Feb 17]; 7(1): 56-61. Disponible en: http://scielo. isciii.es/scielo.php?script=sci_arttext\&pid=S1139$13752004000100006 \& \operatorname{lng}=e s$.

20. Vilas Rivarés A. et al. Análisis del cumplimiento farmacológico de los pacientes en hemodiálisis. Rev Soc Esp Enferm Nefrol, Madrid, v. 4 n. 2 jul. 2006.

21. Arenas MD et al. Challenge of phosphorus control in hemodialysis patients: a problem of adherence? J Nephrol. 2010. [citado 2010 Feb 17]. Disponible en: J Nephrol. 2010 Jan 29. pii: 79597BF2-B665410C-83E5-6128A5E2E98C. 